\title{
Role of Nuclear Motion in Double Ionization of Molecular Hydrogen by a Single Photon
}

\author{
D. A. Horner, ${ }^{1, *}$ W. Vanroose, ${ }^{2}$ T. N. Rescigno, ${ }^{1}$ F. Martín, ${ }^{3}$ and C. W. McCurdy ${ }^{1,4}$ \\ ${ }^{1}$ Lawrence Berkeley National Laboratory, Chemical Sciences, Berkeley, California 94720, USA \\ ${ }^{2}$ Departement Wiskunde-Informatica, Universiteit Antwerpen, 2020 Antwerpen, Belgium \\ ${ }^{3}$ Departamento de Química C-9, Universidad Autónoma de Madrid, 28049 Madrid, Spain \\ ${ }^{4}$ Departments of Applied Science and Chemistry, University of California, Davis, California 95616, USA
}

(Received 26 October 2006; published 12 February 2007)

\begin{abstract}
We examine the origin of recently observed variations with internuclear distance $(R)$ of the fully differential cross sections for double ionization of aligned $\mathrm{H}_{2}$ by absorption of a single photon. Using the results of fully converged numerical solutions of the Schrödinger equation, we show that these variations arise primarily from pronounced differences in the $R$ dependence of the parallel and perpendicular components of the ionization amplitude. We also predict that $R$ dependences should be readily observable in the asymmetry parameter for photodouble ionization, even in experimental measurements that are not differential in the energy sharings between ejected photoelectrons.
\end{abstract}

DOI: 10.1103/PhysRevLett.98.073001

PACS numbers: 33.80.Eh, 31.15.Ar

Double ionization that results from absorption of a single photon by an atom or molecule is exquisitely sensitive to electron correlation in both the initial and final states. Indeed, in the independent electron model, the double ionization probability is zero, which is the consequence of both the exact orthogonality of the atomic or molecular orbitals and the one-body character of the dipole operator. For the case of $\mathrm{H}_{2}$, the simplest molecular target, double photoionization (DPI) is accompanied by a Coulomb explosion of the nuclei, and recent momentum imaging experiments [1-4] in which all four charged particles are measured in coincidence have shown that the correlated motion of the ejected electrons is sensitive to the kinetic energy release (KER) of the dissociating nuclei. The KER, as we verify below, is directly related to the internuclear separation of the target at the moment of photon absorption via the relation $\mathrm{KER}=1 / R$. Interpretation of such experiments requires precise solutions of the Schrödinger equation to unambiguously determine the origin of the observed effects and to guide experimenters in determining which observations are most sensitive to changes in electron correlation with bond distance. We have shown [5] that such precise calculations are possible, with no appeal to models other than the assumed validity of the Born-Oppenheimer approximation in solving the fixed-nuclei Schrödinger equation, and we recently reported the details of fully converged calculations of photodouble ionization of aligned $\mathrm{H}_{2}$ at its equilibrium bond distance [6].

Our purpose in writing this Letter is threefold: (i) to demonstrate that precise calculations can reproduce the strong and surprising variations in the differential cross sections with respect to nuclear KER recently reported by Weber et al. [1,2], (ii) to explain the origin of the observed effects in the fully differential cross sections, and (iii) to use the calculations as a predictive tool to find other geometries and energy sharings where the $R$ dependence of the fully differential cross sections (FDCS) would be more prominently revealed than in current experiments, even after averaging over the finite ranges of ejection angles, molecular orientations, energy sharings, and nuclear kinetic energies that characterize the experimental observations.

To accomplish our first goal of performing precise calculations of the variation of the cross sections with kinetic energy release, our earlier calculations [6] have to be extended to cover the full range of internuclear distance sampled by the molecule in its ground vibrational state. When the photon energy is well above the threshold for double ionization, we can safely employ the BornOppenheimer approximation to separate electronic and nuclear motion. If the rotational temperature of the target gas is low, we can also assume that, following photoabsorption, the nuclei fly apart along a vector that remains stationary (i.e., does not rotate) in the laboratory frame. Under these conditions, we can write the DPI amplitude as

$$
F_{\kappa}\left(\hat{\mathbf{k}}_{1}, \hat{\mathbf{k}}_{2}, \hat{\boldsymbol{\epsilon}}\right)=\int_{0}^{\infty} d R \chi_{\kappa}(R) f\left(\mathbf{k}_{1}, \mathbf{k}_{2}, \hat{\epsilon} ; R\right) \chi_{0}(R),
$$

where $f\left(\mathbf{k}_{1}, \mathbf{k}_{2}, \hat{\boldsymbol{\epsilon}} ; R\right)$ is the amplitude for a photon with polarization $\hat{\epsilon}$ to be absorbed by a fixed-in-space molecule with internuclear separation $R$ and produce photoelectrons with momenta $\mathbf{k}_{1}$ and $\mathbf{k}_{2}$. The functions $\chi_{0}(R)$ and $\chi_{\kappa}(R)$ are the initial (bound) and final (energy-normalized continuum) vibrational wave functions, respectively. Energy conservation (in atomic units):

$$
E_{0}+h \nu=k_{1}^{2} / 2+k_{2}^{2} / 2+\kappa^{2} / 2 \mu
$$

relates the target energy $E_{0}$, photon energy $h \nu$, photo electron energies $k_{1}^{2} / 2$ and $k_{2}^{2} / 2$, and kinetic energy release $\kappa^{2} / 2 \mu$, where $\mu$ is the reduced mass of the nuclei. In the present case, $\chi_{\kappa}(R)$ is simply the repulsive Coulomb function corresponding to two bare protons. 
The fixed-nuclei amplitudes $f\left(\mathbf{k}_{1}, \mathbf{k}_{2}, \hat{\epsilon} ; R\right)$, whose evaluation is the key difficulty in DPI calculations, were obtained by formulating the problem using "exterior complex scaling." The details of that formulation have been thoroughly discussed in Refs. [6,7] and will not be repeated here. We have used finite elements and the discrete variable representation in carrying out these calculations, and the computational details such as grid size, number of basis functions used, and number of partial waves retained, are identical to those used in [6].

The spatial extent of $\chi_{0}(R)$ effectively limits the integration in Eq. (1) to a finite range of $R$ values (FranckCondon interval) slightly larger than $R_{\text {inner }} \leq R \leq R_{\text {outer }}$, which in turn limits the range of observable values of the KER. Since the principal contribution to the integral in Eq. (1) comes from $R$ values near the classical turning point, $R_{\mathrm{tp}}$, on the final ion potential curve, the integral will be small unless $R_{\mathrm{tp}}=1 / \mathrm{KER}$ lies in the range where $\chi_{0}(R)$ is appreciably nonzero. When the photon energy is well above the Franck-Condon threshold, as it is in the cases we report here, the calculations can be simplified by using the method of stationary phase to approximate the integral in Eq. (1). The result [6], called the classical reflection approximation, is equivalent to replacing the nuclear continuum wave function $\chi_{\kappa}(R)$ in Eq. (1) by a $\delta$ function at the classical turning point and thus, in this limit, there is a one-to-one mapping between internuclear distance and KER. With this approximation, the FDCS for DPI, which is differential in the KER $E_{N}$, the photoelectron energy $E_{1}$, and the photoelectron directions $\Omega_{1}$ and $\Omega_{2}$, is given by

$$
\begin{aligned}
\frac{d^{6} \sigma}{d E_{1} d E_{N} d \Omega_{1} d \Omega_{2}}= & \frac{4 \pi^{2}}{\omega c} k_{1} k_{2}\left|F_{\kappa}\left(\hat{\mathbf{k}}_{1}, \hat{\mathbf{k}}_{2}, \hat{\boldsymbol{\epsilon}}\right)\right|^{2} \\
\approx & \frac{4 \pi^{2}}{\omega c} k_{1} k_{2}\left[R_{\mathrm{tp}} \chi_{0}\left(R_{\mathrm{tp}}\right)\right. \\
& \left.\times\left|f\left(\mathbf{k}_{1}, \mathbf{k}_{2}, \hat{\boldsymbol{\epsilon}} ; R_{\mathrm{tp}}\right)\right|\right]^{2} .
\end{aligned}
$$

The reflection approximation was tested by LeRouzo [8] in calculations of the total DPI cross section for $\mathrm{H}_{2}$ and found to be quite accurate. We have verified that the reflection approximation is justified in the present study of the FDCS by using it to compute differential cross sections for three different values of KER and several molecular orientations and energy sharings and comparing with results in which the integral in Eq. (1) was explicitly performed numerically. Differences were typically on the order of a few percent, and in no way affect the conclusions of this study. In order to compare with experiment, the cross sections must be integrated over a finite range of KER and angles, and since the evaluation of the fixed-nuclei amplitude $f\left(\mathbf{k}_{1}, \mathbf{k}_{2}, \hat{\boldsymbol{\epsilon}} ; R\right)$ is computationally intensive this approximation simplifies those calculations considerably. More importantly, our verification of the accuracy of the reflection approximation validates its use by Weber et al. in the original interpretation of their experiments.
We have found that it is essential in comparing with the present generation of DPI experiments on molecules to integrate the theoretical results over the experimental angular and energetic resolutions. For the comparisons shown in Fig. 1, which shows data for a photon energy of $75 \mathrm{eV}$ and equal energy sharing for the two ejected electrons, we averaged the calculated FDCS over the finite ranges of molecular orientation $\left( \pm 24^{\circ}\right)$, photoelectron direction $\left[ \pm 35^{\circ}\right.$ and $\pm 26^{\circ}$ (fixed electron)], energy sharing $( \pm 10 \%)$ and KER $(\sim 2.5 \mathrm{eV})$ described in Ref. [1], and in the corrected experimental results published by those authors in a later erratum [2].

This comparison verifies a key result of the experiments, namely, an apparent rotation in the emission pattern of ejected electrons as the bond distance varies. The effect is most apparent in the full 3D plots of the FDCS. As Weber et al. [2] point out, in the analogous atomic case of helium the light field apparently drives electrons to-

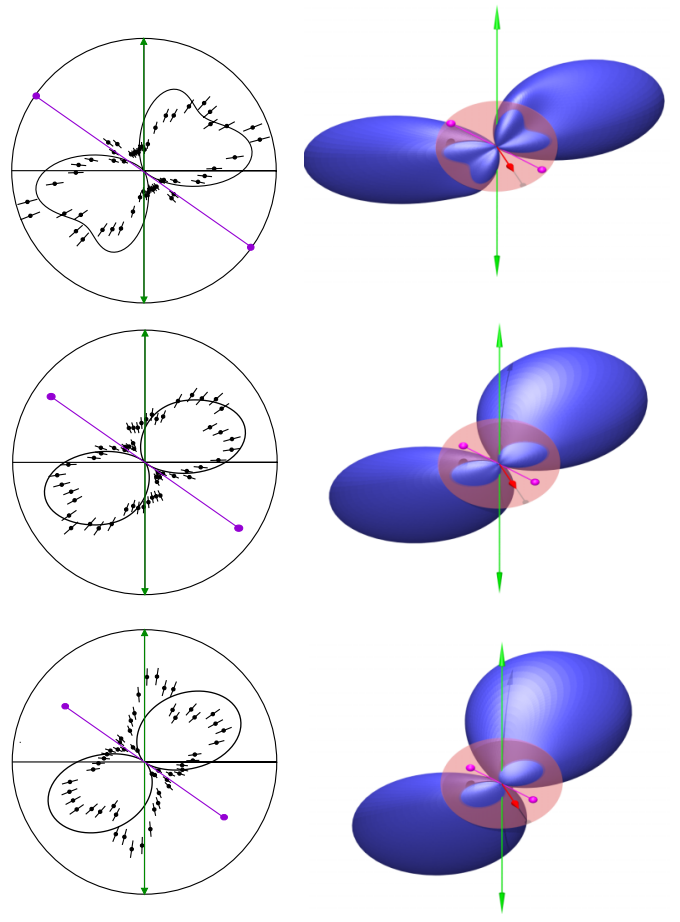

FIG. 1 (color online). FDCS for a case with equal energy sharing between the two electrons. The fixed electron (single arrow) is perpendicular to the plane formed by the molecule and the polarization (vertical double arrow). The molecule is aligned at $55^{\circ}$ to the polarization. The results (top to bottom) are for KER of $0.50-0.60,0.65-0.75$, and $0.75-0.85$ hartrees, respectively. On the left we compare with the relative experimental FDCS (points) of [1,2], in which the panels are internormalized and, for visual clarity, divided by the factor $\int_{R_{1}}^{R_{2}}\left[R \chi_{0}(R)\right]^{2} d R$, where $R_{1}$ and $R_{2}$ are the internuclear distances corresponding to the specified ranges of KER. The theoretical results (solid lines) were integrated over the finite ranges of electron ejection angles, energy sharings, and molecular orientations of the experiment. Right: corresponding full 3D FDCS for KER of 0.55, 0.70, and 0.80 hartrees, not integrated over any experimental acceptance ranges. 
wards a dipole pattern keyed to the axis of polarization, whereas in the geometry of the experiment shown in Fig. 1 the molecule emits the in-plane electron strongly perpendicular to its axis. As the molecule is stretched that effect becomes more pronounced.

As a way to exhibit the origin of this effect we also plotted the FDCS in 3D in Fig. 1. The experiment measures (within its angular resolution) a cut of that 3D figure for each range of KER (internuclear distances) reported. We see the apparent rotation of the FDCS is even more pronounced in the entire 3D representation of the cross section because the experiment is sampling only a thin slice of the front of a distribution that is pushed behind the plane by the repulsion of the measured electron by the fixed electron being ejected out of the plane. The experiments have detected this important effect, but the cuts displayed on the left side of Fig. 1 do not show the majority of the large lobes of the FDCS that are evident in the full 3D panels. The apparent structure in the lower left panel of Fig. 1 is not verified by the calculations, and we speculate it is due to the experimental statistics, but some similar structure appears in both the theory and experiment in the top panel. However, these are minor features of the electron distribution.

The principle question is the following: Why does the FDCS rotate with changing internuclear distance? The FDCS is a coherent sum of contributions to the DPI amplitude from the components of the polarization parallel $\left(\Sigma_{u}\right)$ and perpendicular $\left(\Pi_{u}\right)$ to the molecular axis [6]. The differing magnitudes of those components was observed to cause a strong dependence of the FDCS on molecular orientation [9], as explained in our previous work [5,6] as well as in earlier model calculations [10,11], but it is not immediately obvious how these differences can give rise to an $R$ dependence in the FDCS for a fixed molecular orientation. We can see the relative magnitudes of the $\Sigma_{u}$ and $\Pi_{u}$ components at a glance by integrating the FDCS in Eq. (3) over the directions of the ejected electrons to define the molecular analogy, $d \sigma / d E_{1} d E_{N}$, of the singly differential cross section (SDCS) in the atomic case. (The SDCS is easily obtained by summing the squared moduli of the "reduced" DPI amplitudes [6].) Because of the applicability of the reflection principle, we can plot this quantity as a function of the internuclear distance $R$ with the units of an atomic single differential cross section, leaving out the factor of $\left[R_{\mathrm{tp}} \chi_{0}\left(R_{\mathrm{tp}}\right)\right]^{2}$ from Eq. (3). We do so in the top panel of Fig. 2, and we see a shallow well in the variation of the singly differential cross section as a function of the energy of one electron $E_{1}[6]$ at each value of $R$. However, this plot also shows a surprising result. The contribution of the parallel $(\Sigma)$ component has a minimum in the range of internuclear distances being sampled by the experiment, while the perpendicular (П) component decreases monotonically as $R$ increases.

The angular distribution of the ions (protons) in terms of their ejection angle $\theta_{\text {mol }}$ relative to the polarization vector $\mathrm{d} \sigma / \mathrm{dE}_{1}$
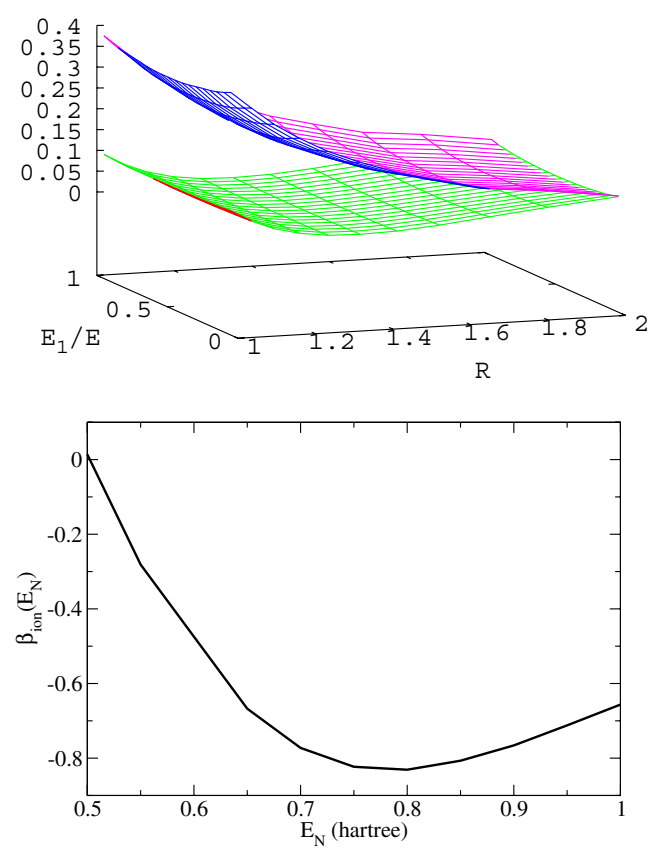

FIG. 2 (color online). Top panel: $\Pi$ (upper surface) and $\Sigma$ (lower surface) contributions to $d \sigma / d E_{1} d E_{n}$, divided by $\left[R_{\mathrm{tp}} \chi_{0}\left(R_{\mathrm{tp}}\right)\right]^{2}$, in units of $\mathrm{kb} / \mathrm{eV}$ (see text), plotted as functions of internuclear distance $R$ in Bohr radii and the ratio $E_{1} / E$, where $E=E_{1}+E_{2}$; bottom panel: $\beta_{\text {ion }}\left(E_{N}\right)$.

is given by the well-known formula [12] $\frac{d^{2} \sigma}{d E_{1} d E_{N}}\left(\theta_{\mathrm{mol}}\right)=$ $\frac{d^{2} \sigma}{d E_{1} d E_{N}}\left[1+\beta_{\text {ion }}\left(E_{1}, E_{N}\right) P_{2}\left(\cos \theta_{\text {mol }}\right) / 4 \pi\right]$. The parameter $\beta_{\text {ion }}\left(E_{1}, E_{N}\right)$ is given by a simple formula that depends on the $\Sigma_{u}$ and $\Pi_{u}$ contributions to $\frac{d \sigma}{d E_{1} d E_{N}}$,

$$
\beta_{\text {ion }}\left(E_{1}, E_{N}\right)=\frac{2\left(\frac{d^{2} \sigma^{(\Sigma)}}{d E_{1} d E_{N}}-\frac{d^{2} \sigma^{(\mathrm{II})}}{d E_{1} d E_{N}}\right)}{\left(\frac{d^{2} \sigma^{(\Sigma)}}{d E_{1} d E_{N}}+2 \frac{d^{2} \sigma^{(\mathrm{II})}}{d E_{1} d E_{N}}\right)} .
$$

The $\beta_{\text {ion }}$ parameter displays a pronounced dependence on $E_{N}$, while showing very little dependence on $E_{1}$. It is therefore plotted in the lower panel of Fig. 2 as a function of only $E_{N}$, where it is defined in terms of cross sections obtained by integrating the $E_{N}$-dependent SDCS over $E_{1}$. The variation in those quantities with $E_{N}$ and, via the reflection approximation, with $R$ is responsible for the rotation of the cross section with changing $R$ in Fig. 1.

We predict here that the dependence of $\beta_{\text {ion }}$ on KER is an observable signature of this effect in an integrated quantity that is easier to measure. The quantity $\beta_{\text {ion }}$, for equal energy sharing but averaged over KER, has been measured by Gisselbrecht et al. [4] who report $\beta_{\text {ion }}=$ $-0.75 \pm 0.1$, while earlier measurements by Kossmann et al. [13] report a value for $\beta_{\text {ion }}$, averaged over both KER and energy sharings, of $-0.68 \pm 0.045$. Both experimental setups are, in principle, able to resolve the KER and measure $\beta$ as a function of $E_{N}$. Our calculations give a value of -0.721 for $\beta_{\text {ion }}$, averaged over KER and energy 


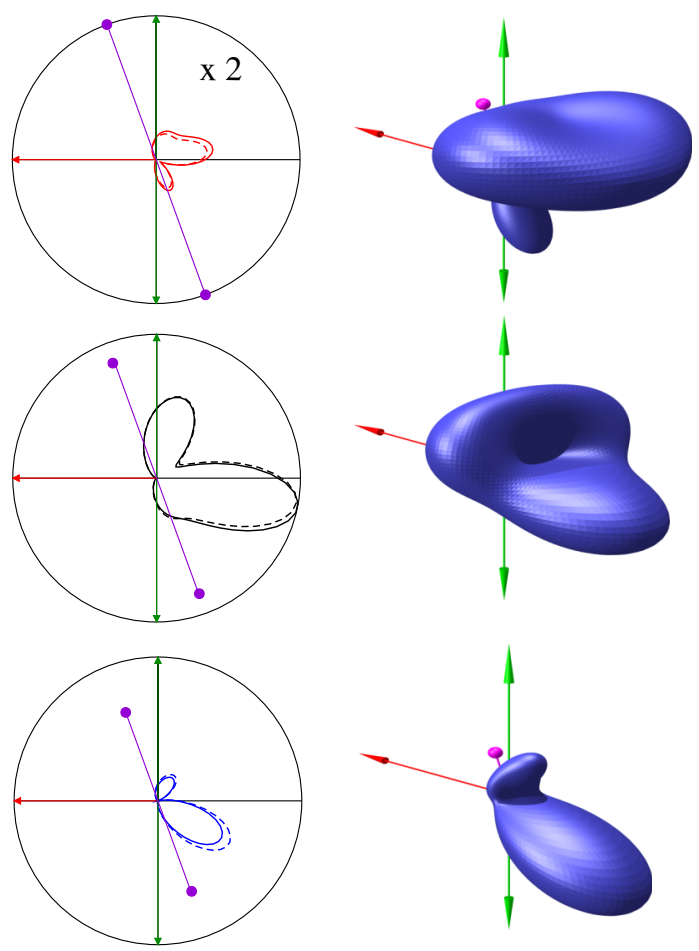

FIG. 3 (color online). FDCS for the case of the molecule aligned at $20^{\circ}$ to the polarization (vertical) and the fixed electron (horizontal arrow pointing left), with $90 \%$ of the available energy, perpendicular to the polarization. The FDCS (top to bottom) is plotted for KER of $0.55,0.70$, and 0.85 hartrees. Right column shows 3D representation, and left column shows a cut in the plane of the molecule, fixed electron, and polarization vectors, where the radius of the circle is $0.16 \mathrm{~b} / \mathrm{eV}^{2} / \mathrm{str}^{2}$. Solid lines are with the reflection approximation; dashed lines are from full integration of Eq. (1).

sharing. The FDCS is of course sensitive not only to the relative magnitudes of the parallel and perpendicular components of the DPI amplitude, but to their phases as well. Nonetheless, a measurement of only the quantity plotted in the bottom panel of Fig. 2, that is, the KER-dependent $\beta$ parameter from cross sections integrated over all electron energy sharings, will display the central cause of the effect observed by Weber et al. in the FDCS.

Having understood the essential physics of that effect, we can use our accurate calculations of the FDCS to find other geometries where these effects are even more prominent. The present generation of experimental measurements observes a cut of the FDCS in the plane of the "measured electron" with the other electron fixed in both direction and energy. The measurements of [1,2] were performed at equal energy sharing. For unequal energy sharing, final state correlation effects are relatively less important and the FDCS should be more sensitive to initial state correlation, which has a strong $R$ dependence. Indeed, we have found that with unequal energy sharing between the two outgoing electrons the $R$ dependence is more evident and that a plane for the measured electron can be found that exhibits that effect quite prominently. Such a geometry is shown in Fig. 3, in which we show (with no averaging) the results of calculations of the FDCS in which the fixed electron has $90 \%$ of the available energy. This is a "coplanar" geometry in which the vectors of the polarization, fixed electron, and observed electron all lie in the same plane. The FDCS in the left column is shown both in the reflection approximation and with the full integration in Eq. (1), thereby verifying the accuracy of the reflection approximation. The right column of that figure shows the three dimensional representation of the FDCS magnified in the top and bottom panels so that the radical change with varying internuclear distance is most visible. We predict that a measurement of the FDCS in this geometry, even integrated over ranges of KER that correspond to the experimental resolution, will show the striking effects of varying internuclear distance more clearly than do the currently published measurements.

In summary, our calculations have allowed us to identify the underlying cause of the pronounced effect on the FDCS of varying internuclear distance observed by Weber et al., propose a more easily observable signature of this phenomenon, and predict geometries in which this essentially molecular effect in DPI is more prominently visible.

Work performed at the Lawrence Berkeley National Laboratory under the auspices of the U.S. Department of Energy under Contract No. DE-AC02-05CH11231 was supported by the U.S. DOE Office of Basic Energy Sciences, Division of Chemical Sciences. F.M. is supported by the DGI (Spain) Project No. BFM2003-00194 and the European COST Action No. D26/0002/02.

*Present address: Los Alamos National Laboratory, Theoretical Division, Los Alamos, NM 87545, USA.

[1] T. Weber et al., Nature (London) 431, 437 (2004).

[2] T. Weber et al., Nature (London) 443, 1014 (2006).

[3] T. Weber, Ph.D. thesis, Institut fuer Kernphysik, Frankfurt, 2003, available at http://hsb.uni-frankfurt.de/web/ publications/diplom_doktor/.

[4] M. Gisselbrecht et al., Phys. Rev. Lett. 96, 153002 (2006).

[5] W. Vanroose, F. Martín, T. N. Rescigno, and C.W. McCurdy, Science 310, 1787 (2005).

[6] W. Vanroose, D. A. Horner, F. Martín, T. N. Rescigno, and C. W. McCurdy, Phys. Rev. A 74, 052702 (2006).

[7] C.W. McCurdy, M. Baertschy, and T.N. Rescigno, J. Phys. B 37, R137 (2004).

[8] H. LeRouzo, Phys. Rev. A 37, 1512 (1988).

[9] Th. Weber et al., Phys. Rev. Lett. 92, 163001 (2004).

[10] T. J. Reddish and J. M. Feagin, J. Phys. B 32, 2473 (1999).

[11] M. Walter and J. Briggs, J. Phys. B 32, 2487 (1999).

[12] J. L. Dehmer and D. Dill, Phys. Rev. A 18, 164 (1978).

[13] H. Kossmann, O. Schwarzkopf, B. Kämmerling, and V. Schmidt, Phys. Rev. Lett. 63, 2040 (1989). 